\title{
Experimental Status of Gravitational-Strength Forces in the Sub-Centimeter Regime
}

\author{
J. C. Long, H. W. Chan, and J. C. Price \\ Department of Physics - CML, University of Colorado, Boulder CO 80309 USA
}

(August 30, 2018)

\begin{abstract}
We review the experimental constraints on additional macroscopic Yukawa forces for interaction ranges below $1 \mathrm{~cm}$, and summarize several theoretical predictions of new forces in this region. An experiment using $1 \mathrm{kHz}$ mechanical oscillators as test masses should be sensitive to much of the parameter space covered by the predictions.
\end{abstract}

04.80.-y, 11.30.Pb, 14.80.Mz

Typeset using REVTEX 


\section{INTRODUCTION}

Experimental searches for a deviation from Newton's law of universal gravitation have received a great deal of attention over the past three decades. Motivation has come from reports of experimental anomalies, and from new theoretical predictions. Searches have involved a great variety of experiments on the planetary, lunar, geological, and laboratory scales. A recent review by Fischbach and Talmadge [1] shows that new forces with a strength weaker than or comparable to gravity have been excluded over distances ranging between 1 and $10^{17} \mathrm{~cm}$. Experiments have only marginally explored the distance range under 1 $\mathrm{cm}$, however, and there is little knowledge of gravity itself in this range [2]. Furthermore, the sub-centimeter region is of increasing experimental interest, given a number of recent

predictions of new forces from modern theories which attempt to unify gravity with the strong and electroweak interactions.

In this paper we present a review of the existing experimental limits and some of the recent theoretical predictions. We then describe an experiment which we expect to be sensitive to gravity at distances as short as $50 \mu \mathrm{m}$, and to much of the parameter space for the new predictions.

\section{CURRENT LIMITS}

Experimental results from searches for new long-range forces of gravitational strength have been parameterized in several ways [3]. The Yukawa interaction is most commonly used, and we adopt it here. The potential due to gravity and an additional Yukawa interaction between two macroscopic objects may be written

$$
V(r)=-\int d r_{1} \int d r_{2} \frac{G \rho_{1}\left(r_{1}\right) \rho_{2}\left(r_{2}\right)}{r_{12}}\left[1+\alpha \exp \left(-r_{12} / \lambda\right)\right]
$$

where $G$ is the Newtonian gravitational constant, $r_{12}$ is the distance between $r_{1}$ and $r_{2}, \rho_{1}\left(r_{1}\right)$ and $\rho_{2}\left(r_{2}\right)$ are mass densities, $\alpha$ is the strength of the Yukawa force relative to gravity, and 
$\lambda$ is the range. If, as is likely, the Yukawa forces couple to quantities other than mass, then $\alpha$ will have a slight composition dependence [4], which we do not consider.

The current experimental limits on $\alpha$, for Yukawa ranges between $1 \mu \mathrm{m}$ and $1 \mathrm{~cm}$, are illustrated in Fig. 1, together with the limit anticipated from the experiment outlined in Sec. IV below. Also indicated are specific predictions for new long-range forces, described in Sec. [II. The region above and to the right of the bold curves is excluded by experiments falling into two general categories: classical gravity measurements and Casimir force measurements.

\section{A. Classical Gravity Experiments}

For values of $\lambda$ greater than $100 \mu \mathrm{m}$, the most stringent limits on $\alpha$ can be obtained from torsion balance experiments designed to test the gravitational inverse-square law. Well before the interest in gravitational measurements brought on by suggestions of a "fifth force," the report of a possible deviation in a measurement by Long [5] motivated several similar experimental programs (see, for example, Refs. 66 8]) which have apparently ruled out any evidence for the anomaly [9].

One such experiment is the Irvine 2-5 cm null measurement [10], which yields the best limits in the range near $1 \mathrm{~cm}$. This experiment used a torsion balance to measure the force on a test mass suspended inside a hollow cylinder, a non-zero result for which would have been evidence for an anomalous force. After an exhaustive consideration of possible systematic effects, and careful analysis of inhomogeneity in the cylinder, the authors derived the limit curve reproduced in Fig. 1, corresponding to a force sensitivity of about $4 \times 10^{-10}$ dyne. Only the curve corresponding to the (more sensitive) limiting case of a hypothesized attractive Yukawa force $(\alpha>0)$ is shown.

Below the range of a few millimeters, the most stringent limits may be obtained from the torsion balance experiment of Mitrofanov and Ponomareva [11]. This experiment used a dynamic-resonant technique to distinguish the gravitational interaction of the test masses 
from backgrounds. A test mass, initially spaced $3.8 \mathrm{~mm}$ perpendicular to one end of the balance, was moved synchronously with the oscillations of the balance. The authors were able to achieve a sensitivity of about $3 \times 10^{-10}$ dyne, for integration times of about $10^{3} \mathrm{~s}$. The ratio of the forces measured at $3.8 \mathrm{~mm}$ and $6.5 \mathrm{~mm}$ was used to obtain a curve similar to that shown in Fig. 11.

We have re-analyzed the data of this experiment, using Eq. 7 of Ref. 11, to extend the limit to shorter distances than those considered by the authors. The resulting curve is shown in Fig. 1. The $1 \sigma$ errors on the test mass separations, apparently not taken into account in the original report, are added in quadrature to the $1 \sigma$ errors on the measured force ratio, so that the limit we derive is slightly less sensitive than that reported in Ref. 11. We have not illustrated the cases for the separate hypotheses of attractive and repulsive forces, which have little effect on the results.

Other inverse-square law and Cavendish experiments have been performed with intermass separations on the order of $1 \mathrm{~cm}$, including that of the Tokyo group [12. None, however, reach stronger limits in the range below $1 \mathrm{~cm}$. The Rot-Wash experiment, which uses a $2620 \mathrm{~kg}$ depleted uranium attractor rotated around a torsion balance with lead and copper test masses, yields a limit nearly competitive with the Irvine result at $1 \mathrm{~cm} \llbracket 13 \rrbracket$. This assumes a Yukawa force which couples to mass; the use of uranium makes this experiment especially sensitive to composition-dependent forces. Assuming a coupling to $N-Z$, the Rot-Wash experiment has set a limit 10 times more stringent than the Irvine experiment at $1 \mathrm{~cm}$.

\section{B. Casimir Force Experiments}

In 1948, H. G. B. Casimir showed that a weak attractive force should be present between a set of flat, parallel conducting plates, due to the zero-point fluctuations of the electromagnetic field [14]. This force is given by

$$
F_{c}=\frac{\pi^{2}}{240} \frac{\hbar c}{r^{4}} A
$$


where $r$ is the separation and $A$ is the area. A similar effect, called the retarded Van der Waals force, occurs with dielectric plates and is given by an expression which differs from Eq. 2 only by a numerical factor of order unity [15].

For a given value of $\lambda$, the most stringent limits on $\alpha$ can be obtained from Casimir and Van der Waals force data taken with mass separations of order $\lambda$, given the strong dependence of Eq. 2 on $r$. These experiments usually investigate separation distances well below $1 \mathrm{~mm}$. In practice, the best limits to date are derived from measurements of the force between a flat plate and a spherical shell, which varies as $r^{-3}$. The experiment by Derjaguin, Abrikosova, and Lifshitz [16] done in 1956 provided the most sensitive limits in the range less than $100 \mu \mathrm{m}$ for 40 years, until the recent measurement by Lamoreaux [17.

This experiment measured the attraction between a flat, metal-plated quartz disk and a metal-plated spherical lens separated by distances ranging from about 0.5 to $10 \mu \mathrm{m}$. Using data from this experiment, we have derived the limits presented in Fig. 1. For several values of $\lambda$ in the range 1-100 $\mu \mathrm{m}$, a least-squares fit is made with the data in the measured force plot (Fig. 3, top, of Ref. 17), to the expression

$$
F_{i}^{m}=F_{c}^{T}\left(a_{i}+a_{0}\right)+\frac{\beta}{a_{i}+a_{0}}+b+F_{Y}\left[\alpha, \lambda, \rho_{j}, d_{j},\left(a_{i}+a_{0}\right)\right] .
$$

This is based on Eq. 7 of Ref. 17, where the first three terms are the Casimir, electromagnetic, and constant terms used by Lamoreaux to fit the data (with the same fit parameters $a_{0}, \beta$, and $b)$. We have added a Yukawa term $F_{Y}\left[\alpha, \lambda, \rho_{j}, d_{j},\left(a_{i}+a_{0}\right)\right]$, in which $\alpha$ is taken as an additional fit parameter. The Yukawa term is a function of the densities $\rho_{j}$ and thicknesses $d_{j}$ of the gold, copper, and quartz comprising the Casimir test plates [18].

For each value of $\lambda$ investigated, $\alpha$ is then fixed at various values within a range of the fit value, and the fit is performed again. The $1 \sigma$ acceptance region in $\alpha$ is then taken to be all the points in the $(\alpha, \lambda)$ parameter space which have a $\chi^{2} /$ d.o.f. within 1.0 of the minimum value. This region, bounded above by the curve in Fig. 1, is roughly one order of magnitude more stringent than the Derjaguin limit over the entire range shown, for positive values of $\alpha$ [19]. It is very important to note that the measured force plot presumably represents less 
than one percent of the total data set [20]. It is therefore likely that the limits attainable from a complete re-analysis of this experiment are at least another order of magnitude more sensitive than those presented in Fig. 11.

\section{Other Limits}

Limits on new forces in the range considered here have been obtained in experiments on other systems, but they are not sensitive enough to compete with the limits shown in Fig. 1. Bracci, Fiorentini, and Tripiccione 21] set limits on long-range nucleon-nucleon interactions from a re-analysis of a variety of experiments. In addition to macroscopic systems, they consider the spectroscopy of hydrogenic and mesic molecules, antiprotonic atoms, and data from low energy nucleon-lead and nucleon-nucleon scattering experiments. The limits on the coupling strength derived from these elementary systems would have to improve by many orders of magnitude in order to appear in Fig. 1. Leeb and Schmiedmayer have derived similar constraints from neutron scattering experiments below $10 \mathrm{keV}$, and from neutron

optics experiments [22]. Studying the difference between scattering lengths measured in these two classes of experiments, they extract limits on the size and range of an additional Yukawa term in the neutron-nucleus interaction. While more sensitive than those obtained in Ref. 21, these limits are again many orders of magnitude too weak to appear in Fig. 11.

In general, since the electrostatic force between protons is $10^{36}$ times stronger than the gravitational force, no experiment on an elementary system which is dominated by electromagnetic effects can be expected to provide limits strong enough in this range to compete with macroscopic measurements [23].

\section{THEORETICAL PREDICTIONS}

As indicated in Fig. 1, current experimental limits allow for the existence of forces many orders of magnitude stronger than gravity in the range below one millimeter. Predictions of new forces - usually involving very light bosons - have arisen in many contexts, from the 
early notion of Lee and Yang of a field coupled to baryon number [24], to modern ideas connected with string theory. For experiments below one millimeter, several well-motivated predictions can be addressed.

Many modern predictions arise in theories which attempt to describe gravitation and the other fundamental interactions in a single framework. The mass of the light boson is typically given by [幽:

$$
m \approx M_{P}\left(\frac{M}{M_{P}}\right)^{n}
$$

where $M_{P} \approx 10^{19} \mathrm{GeV}$ is the Planck mass (or string scale), $M$ is a mass scale connected with the known elementary particles, and $n$ is a small integer. For $n=2$, the choice of $M=10$ TeV yields $m=10^{-2} \mathrm{eV}$, corresponding to a Yukawa force with a range of $\lambda=20 \mu \mathrm{m}$.

An interesting class of predictions arises in superstring theories in which supersymmetry breaking occurs at low energies (less than about $100 \mathrm{TeV}$ ). These theories are of interest as a way to address the supersymmetry flavor problem. Superstring models generally contain gravitationally-coupled scalars called moduli which are massless at the string scale. Dimopoulos and Giudice consider a "gauge-mediated" model where the moduli acquire mass only from supersymmetry breaking, which they suppose to occur at 10-100 TeV [25]. This corresponds to Eq. 4 with $M=10-100 \mathrm{TeV}$ and $n=2$. The predicted strength and range of the modulus forces associated with the down quark, strange quark, and the gluon are shown in Fig. 1. Each prediction covers a region in the $(\alpha, \lambda)$ plane because of uncertainties in the model parameters. The predicted strengths are larger than gravity by several orders of magnitude, and the ranges are several orders larger than would be expected from Eq. $甘$. Similar scenarios have been discussed previously by Banks, Berkooz, and Steinhardt [26].

Antoniadis, Dimopoulos, and Dvali have presented a related theory in which supersymmetry breaking occurs via Scherk-Schwarz compactification at the weak scale $(M \approx 1 \mathrm{TeV})$ [27]. The authors show that theories involving this mechanism are likely to give rise to at least one scalar particle, the radius modulus, with a Compton wavelength corresponding to the size of the compact dimension. In the model considered, the radius modulus has a 
Compton wavelength in the sub-millimeter range, and mediates a force with strength $1 / 3$ that of gravity, as shown in Fig 1 .

Other light scalars with stronger couplings are also possible. The dilaton of string theory couples to matter with a strength roughly 80 times that of gravity [28,29]. The mass of the dilaton depends on unknown physics. Dimopoulos and Giudice suggest a dilaton mass of about $10 \mathrm{eV}$ for their scenario, but other authors take the dilaton mass to be an unknown parameter [30]. The existing limits shown in Fig. [1 exclude a mass less than $10^{-3} \mathrm{eV}$.

The axion is a light pseudoscalar boson motivated by the strong CP problem of the standard model. Laboratory experiments and astrophysical bounds have left an allowed window for the axion mass of $10^{-6}$ to $10^{-3} \mathrm{eV}$, or $\lambda=20 \mathrm{~cm}-0.2 \mathrm{~mm}$ [31]. However, the coupling $\alpha$ of the associated long-range force between unpolarized nucleons has been constrained, by recent measurements of the neutron electric dipole moment, to the region of the $(\alpha, \lambda)$ plane shown in Fig. 1. This is probably outside the reach of the experiment described in Sec. [V], but could be a possible goal for the future [32]. Moody and Wilczek [33 have argued that centimeter-scale experiments with polarized bodies would be more promising, and several attempts in this direction have been made [34 37.

Still other models are motivated by cosmological observations. The oscillating-G cosmology of Steinhardt and Will involves a massive Brans-Dicke field which is left oscillating at high frequency after inflation [38]. Yukawa corrections to the Newtonian potential are predicted, and useful bounds on a product of the parameters of the model are found when these predictions are compared to experiments. The experiment described below would improve these bounds by a factor of 600 [39]. A recent model due to Beane is based on more general assumptions. The author argues that if all experimentally observable phenomena can be described in terms of effective field theories, then the observed smallness of the cosmological constant implies the existence of new quanta [40]. Furthermore, if a substantial component of the energy density of the universe in the present epoch is due to vacuum energy, then the range of the new quanta should be approximately $100 \mu \mathrm{m}$, as shown in Fig. [1. R. Sundrum develops these ideas further and proposes a specific mechanism that could provide a solution 
to the cosmological constant problem [41]. In this theory, the new quanta are comprised of light gravitational strings. The size of the cosmological constant is set by the scale of these strings (as opposed to the standard model scale), below which the behavior of gravitational forces is expected to be quite different. Consistency with current cosmological and laboratory constraints suggests this scale to be between $10 \mu \mathrm{m}$ and $1 \mathrm{~cm}$.

Finally, we mention a theory which does not necessarily make recourse to new light particles. In a recent discussion, Arkani-Hamed, Dimopoulos, and Dvali propose a theory in which gravitational and gauge interactions are united at the weak scale, which is taken to be the only fundamental short scale in nature [42]. This model does not rely on supersymmetry and is of interest as a solution to the hierarchy problem. The relative weakness of the gravitational interaction is a consequence of $n \geq 2$ new compact dimensions which are large compared to the weak scale, and in which gravitons propagate freely but the standard model fields do not. For the smallest number of new dimensions yet to be ruled out by

experiment $(n=2)$, the size of the new dimensions is in the millimeter region. Gravitational measurements in this regime would be sensitive to potentials dictated by Gauss' law in $(4+n)$ dimensions, or gravitational forces varying as $r^{-4}$.

From this discussion it is clear that forces of gravitational strength are playing an increasingly important theoretical role, especially since the advent of string theory. There is ample motivation to push to the shortest ranges possible, where macroscopic experiments might provide a rare window on physics at the Planck scale.

\section{SHORT-RANGE MEASUREMENT WITH MECHANICAL OSCILLATORS}

As the length scale $L$ of an experiment decreases, the gravitational attraction decreases as $L^{4}$. It therefore becomes difficult at shorter distances to distinguish the Newtonian force between the test masses from the background forces. Surface forces are especially troublesome, because they increase rapidly at small separations. The most sensitive experiments in the centimeter range to date have used torsion balances. Klimchitskaya, et al., have 
proposed to use a torsional pendulum inside a rotating steel sphere with a non-concentric cavity, and they expect to improve the current limits by roughly an order of magnitude in the range above $100 \mu \mathrm{m}$ [43].

Another approach involves the use of small mechanical oscillators for the experimental test masses. The experiment of Carugno, Fontana, Onofrio, and Rizzo measured the force between a small copper mass and a steel resonator driven at 30 and $1.66 \times 10^{4} \mathrm{~Hz}$, respectively [44. Using a heterodyne detection technique, this experiment was able to set limits on new macroscopic forces between, for example, $\alpha=1 \times 10^{10}$ at $\lambda=2 \times 10^{-5} \mathrm{~m}$ and $\alpha=8 \times 10^{7}$ at $\lambda=2 \times 10^{-4} \mathrm{~m}$. These limits are roughly two orders of magnitude weaker than those shown in Fig. 1, however, the authors hope to improve their sensitivity by as much as five orders of magnitude using fiber optic techniques.

Our own design is illustrated in Fig. 2. Small mechanical resonators are used for both the source mass and force detector. The source mass is driven at the resonant frequency of a detector torsional mode, nominally $1 \mathrm{kHz}$. In the resonant mode of interest, the two rectangular sections of the detector counter-rotate. The amplitude of motion is large for the smaller section immediately above the source mass, while the larger section is nearly stationary. This double torsional arrangement provides a degree of isolation from the oscillator mounting, which decreases the mode damping 445.

The principal advantage of this high-frequency resonant method is that seismic and other vibrational backgrounds can be easily filtered in the kilohertz range. Vibration isolation is provided by supporting the masses from stacks of brass disks connected by steel wires [46,47]. Other backgrounds include electrostatic forces, which are suppressed with a gold-plated shield between the masses, and magnetic forces due to both external fields and contamination. Background due to the Casimir effect is comparable to electromagnetic effects only for mass separations below $1 \mu \mathrm{m}$, roughly $1 \%$ of the anticipated minimum gap.

If these backgrounds can be sufficiently reduced, the dominant source of noise will be thermal noise due to dissipation in the detector oscillator. Based on this limitation, we calculate the expected sensitivity of the experiment to a new Yukawa-type interaction. 
The Yukawa force between parallel plates separated by a distance $d(t)$ is given by:

$$
F_{Y}(t)=2 \pi \alpha G \rho_{s} \rho_{d} A \lambda^{2} \exp (-d(t) / \lambda)\left[1-\exp \left(-t_{s} / \lambda\right)\right]\left[1-\exp \left(-t_{d} / \lambda\right)\right]
$$

where $\rho_{s}$ and $\rho_{d}$ are the source and detector mass densities, and $t_{s}$ and $t_{d}$ are the thicknesses. This would be an exact expression if either plate had area $A$ and the other had infinite area, but for the real geometry there are small edge corrections. Neglecting these effects, and assuming the geometry in Fig. $2 \mathrm{~b}$, the torque exerted on the detector (the smaller rectangle only) via a Yukawa interaction with the source is given by:

$$
N_{Y}(t)=\pi \alpha G \rho_{s} \rho_{d} A_{d} R \lambda^{2} \exp (-d(t) / \lambda)\left[1-\exp \left(-t_{s} / \lambda\right)\right]\left[1-\exp \left(-t_{d} / \lambda\right)\right]
$$

where $A_{d}$ is the area of the detector above the source mass, and $R$ is the distance between the edge of the detector and the torsion axis.

The read-out signal from the transducer varies with the amplitude of the applied torque. The source mass is driven at the detector resonant frequency $\omega_{0}$, and only the amplitude of the signal torque associated with this frequency is effective in driving the detector. From Fourier analysis, this amplitude is:

$$
\left|N_{Y}\left(\omega_{0}\right)\right|=2 \pi \alpha G \rho_{s} \rho_{d} A_{d} R \lambda^{2} I_{1}\left(d_{0} / \lambda\right) \exp (-\bar{d} / \lambda)\left[1-\exp \left(-t_{s} / \lambda\right)\right]\left[1-\exp \left(-t_{d} / \lambda\right)\right]
$$

where $I_{1}\left(d_{0} / \lambda\right)$ is the modified Bessel function. Here we have used $d(t)=\bar{d}+d_{0} \sin \omega_{0} t$, where $d_{0}=\left(d_{\max }-d_{\min }\right) / 2$ is the amplitude of the source mass motion and $\bar{d}=\left(d_{\max }+d_{\min }\right) / 2$ is the average source-detector gap.

The rms thermal noise torque about the central axis is found from the mechanical Nyquist theorem to be:

$$
N_{T}=\sqrt{\frac{4 k T}{\tau}\left(\frac{m R^{2} \omega_{0}}{3 Q}\right)},
$$

where $m$ is the mass of the small rectangular section of the detector oscillator, $Q$ is the detector quality factor, and $\tau$ is the measurement integration time. 
The signal-to-noise ratio is then simply the ratio of equations 7 and 8 . Setting the SNR equal to unity and solving for $\alpha$, we obtain the following result for the limit which the experiment may set on the Yukawa strength for each value of the range:

$$
\alpha=\frac{2}{\pi \sqrt{3}} \frac{1}{G \rho_{s} \rho_{d} \lambda^{2} A_{d}}\left(\frac{1}{\epsilon}\right) \sqrt{\frac{k T m \omega_{0}}{Q \tau}}
$$

The efficiency factor $\epsilon$, which is of order unity for an optimized geometry, is given by:

$$
\epsilon=2 I_{1}\left(d_{0} / \lambda\right) \exp (-\bar{d} / \lambda)\left[1-\exp \left(-t_{s} / \lambda\right)\right]\left[1-\exp \left(-t_{d} / \lambda\right)\right]
$$

The bold curve in Fig. 1, representing the expected sensitivity of the experiment, is calculated using Eqs. 9 and 10 and the parameters in Table $\mathbb{1}$. For each value of $\lambda$, the

maximum separation $d_{\max }$ is chosen so as to maximize the efficiency $\epsilon$. The maximum separation is allowed to vary between the anticipated upper limit of $1 \mathrm{~mm}$ and a lower limit of $100 \mu \mathrm{m}$, below which the backgrounds are expected to dominate. From the limit curve in Fig. 1, we expect the experiment to cover roughly 10 square decades of the $(\alpha, \lambda)$ parameter space previously unexplored below $1 \mathrm{~mm}$.

\section{CONCLUSION}

Torsion balance experiments to the present have been sensitive to gravitational-strength forces down to millimeter distance scales. While very precise Casimir force measurements have recently been carried out and set new limits on effects in the range below $100 \mu \mathrm{m}$, our calculations reveal that these experiments still allow for forces several million times stronger than gravity in this region. Experiments of gravitational sensitivity in this range have become all the more imperative with several recent theoretical predictions of new forces, motivated especially by advances in string theory. The experiment described in this paper, which uses high-frequency mechanical oscillators for the test masses, is expected to reach gravitational strength down to approximately $50 \mu \mathrm{m}$, and to be sensitive to much of the parameter space for these new predictions. 


\section{ACKNOWLEDGMENTS}

We would like to thank S. K. Lamoreaux for useful discussions of his Casimir force data, G. Giudice for an explanation of the superstring models, C. Dembowski for his work in our laboratory, and L. DiLella and R. Onofrio for useful comments. We acknowledge T. Banks, R. Sundrum and K. T. Mahanthappa for alerting us to Refs. 26, 41, and 42, respectively. This work is supported by NSF grant PHY97-22098 and the NSF-REU Program at the

University of Colorado. H. C. acknowledges the support of the McNair Program at the University of Colorado. 


\section{REFERENCES}

[1] E. Fischbach and C. Talmadge, Ten Years of the Fifth Force, in: Proceedings of the XXXI Rencontres de Moriond (Les Arcs, 20-27 January 1996), ed. R. Ansari et al. (Editions Frontieres, Gif-Sur-Yvette, 1996), p. 443.

[2] J. C. Price, in: Proceedings of the International Symposium on Experimental Gravitational Physics, ed. P. Michelson, H. En-ke, and G. Pizzella (D. Reidel, Dordrecht, 1987).

[3] A. H. Cook, Contemp. Phys. 282 (1987) 159-175.

[4] Y. Fujii, Int. J. Mod. Phys. A 620 (1991) 3505.

[5] D. R. Long, Nature 260 (1976) 417.

[6] H. A. Chan, M. V. Moody, H. J. Paik, Phys. Rev. Lett. 49 (1982) 1745.

[7] Y. Ogawa, K. Tsubono, H. Hirakawa, Phys Rev. D 26 (1982) 729.

[8] Y. T. Chen, A. H. Cook, A. J. F. Metherell, Proc. R. Soc. A 394 (1984) 47.

[9] We note, however, the precision torsion balance measurement by Michaelis, H. Haars, and R. Augustin, Metrologia 32 (1995/96) 267. This experiment observed a systematically high value for the Newtonian gravitational constant over roughly the same range as Long's experiment, but did not find evidence for any distance dependence of this result. A subsequent measurement in the same range [J. Schurr et. al., Phys. Rev. Lett. 806 (1998) 1142] reports no evidence of an anomaly.

[10] J. K. Hoskins, R. D. Newman, R. Spero, and J. Schultz, Phys. Rev D 3212 (1985) 3084.

[11] V. P. Mitrofanov and O. I. Ponomareva, Sov. Phys. JETP 6710 (1988) 1963.

[12] N. Mio, K. Tsubono, H. Hirakawa, Phys. Rev. D 36 (1987) 2321. 
[13] J. H. Gundlach, G. L. Smith, E. G. Adelberger, B. R. Heckel, H. E. Swanson, Phys. Rev. Lett. 7813 (1997) 2523.

[14] H. G. B. Casimir, Proc. Kon. Ned. Akad. Wetenschap., ser. B 51 (1948) 793.

[15] B. Chu, Molecular Forces, based on the Baker Lectures of P. W. Debye (Interscience, New York, 1967), ch. 5.

[16] B. V. Derjaguin, I. I. Abrikosova, E. M. Lifshitz, Quarterly Reviews (London, The Chemical Society), 10 (1956) 295.

[17] S. K. Lamoreaux, Phys. Rev. Lett. 781 (1997) 5.

[18] The actual form of $F_{Y}$ contains 17 terms of the form $F_{Y}=4 \pi G \rho_{1} \rho_{2} R \alpha \lambda^{3} \exp \left[-\left(a_{i}+a_{0}\right) / \lambda\right] \exp -d_{12} / \lambda$. This is the expression for the Yukawa force between a solid sphere and an infinite plate or disk, where $G$ is Newton's Gravitational constant, $\rho_{1}$ and $\rho_{2}$ are mass densities, $R$ is the sphere radius, $\left(a_{i}+a_{0}\right)$ is the absolute disk-sphere separation, and $d_{12}$ is an additional separation due to the thicknesses of the Casimir plate materials. Terms of this form are summed in a manner so as to obtain, by superposition, the force between the composite lens and disk used in the experiment.

[19] For negative values of $\alpha$, the limits are approximately the same as those of the Derjaguin experiment. The limits derived from the Mitrofanov and Irvine experiments for $\alpha<0$, as reported in Refs. 10 and 11, are weaker than the limits for $\alpha>0$ only by small numerical factors and are therefore not shown in Fig. 1.

[20] Ref. 17 includes summary and residual plots which demonstrate a convincing signal for the Casimir effect using the entire data set. The data in these plots, however, are adjusted for absolute disk-sphere separation and electromagnetic background as derived from the 3-component fit (Eq. 7 of Ref. 17) to the measured force data. Since the 3 components (Casimir, electromagnetic, and a constant term) do not include a Yukawa 
term, which at current limits can be of the same order as the largest of any of the other three, the summary data cannot be used consistently to derive new force limits. This limits our analysis to a re-fit of the small sub-sample of data presented in the measured (unadjusted) force plot.

[21] L. Bracci, G. Fiorentini, and R. Trippicione, Nuclear Physics B217 (1983) 215.

[22] H. Leeb and J. Schmiedmayer, Phys. Rev. Lett. 6810 (1992) 1472.

[23] We note that elementary systems may still be the most effective probes for certain new long-range effects. Barshay et al. [Mod. Phys. Lett. A 920 (1994) 1835] show that highenergy proton-proton elastic scattering experiments have yet to rule out forces mediated by two-gluon exchange, which they consider to couple with a strength of at least a few percent of the Coulomb interaction and vary as $r^{-6}$

[24] T. D. Lee and C. N. Yang, Phys. Rev. 98 (1955) 1501.

[25] S. Dimopoulos and G. Giudice, Phys. Lett. B 379 (1996) 105.

[26] T. Banks, M. Berkooz, and P. J. Steinhardt, Phys. Rev. D 52 (1995) 705.

[27] I. Antoniadis, S. Dimopoulos, and G. Dvali, Nuclear Physics B 516 (1998) 70.

[28] T. R. Taylor and G. Veneziano, Phys. Lett. B 213 (1988) 450.

[29] J. Ellis et. al., Phys Lett. B. 228 (1989) 264.

[30] M. Gasperini, Phys Rev. D 50 (1994) 2519.

[31] M. S. Turner, Physics Reports 197 (1990) 67.

[32] R. Barbieri, A. Romanino, A. Strumina, Phys. Lett. B 387 (1996) 310.

[33] J. E. Moody and F. Wilczek, Phys Rev. D 30 (1984) 130.

[34] A. N. Youdin, D. Krause, K. Jagannathan, L. R. Hunter, S. K. Lamoreaux, Phys. Rev. Lett. 77 (1996) 2170. 
[35] R. C. Ritter, L. I. Winkler, G. T. Gillies, Phys. Rev. Lett. 706 (1993) 701.

[36] D. J. Wineland, J. J. Bolinger, D. J. Heinzen, W. M. Itano, M. G. Raizen, Phys. Rev. Lett. 67 (1991) 1735.

[37] B. J. Venema, P. K. Majumder, S. K. Lamoreaux, B. R. Heckel, E. N. Fortson, Phys. Rev. Lett. 68 (1992) 135.

[38] P. J. Steinhardt and C. M. Will, Phys Rev D 52 (1995) 628.

[39] C. M. Will, private communication.

[40] S. R. Beane, Gen. Rel. Grav. 29 (1997) 945.

[41] R. Sundrum, CERN preprint hep-ph/9708329.

[42] N. Arkani-Hamed, S. Dimopoulos, and G. Dvali, Phys. Lett. B 429 (1998) 263.

[43] G. L. Klimchitskaya et al., Int. J. Mod. Phys. A 128 (1997) 1465.

[44] G. Carugno, Z. Fontana, R. Onofrio, and C. Rizzo, Phys. Rev. D 55 (1997) 6591.

[45] R. N. Kleiman, G. K. Kaminsky, J. D. Reppy, R. Pindak, and D. J. Bishop, Rev. Sci. Inst. 56 (1985) 2088.

[46] W. M. Fairbank, et al., in: Proceedings of the Second Marcel Grossmann Meeting on General Relativity, ed. R. Ruffini (Amsterdam, North-Holland, 1982).

[47] R. Taber, Attenuation of Mechanical Vibrations in Wires (Stanford University, 1979), unpublished. 
FIG. 1. Parameter space for Yukawa-type forces in which the strength relative to gravity $(\alpha)$ is plotted versus the range $(\lambda)$. Limit curves from experiments defining the excluded region are shown (bold lines) along with the anticipated sensitivity of the proposed experiment, as well as specific predictions for new long range forces (fine and dashed lines).

FIG. 2. a) principal components of the apparatus; b) cross-section of central region showing dimensions used in calculation of experimental sensitivity. 
TABLE I. Parameters used for experimental sensitivity calculation.

\begin{tabular}{lr}
\hline \hline \multicolumn{1}{c}{ Parameter } & Value \\
\hline Signal Frequency, $\omega / 2 \pi$ & $1 \mathrm{kHz}$ \\
Quality Factor, $Q$ & $1 \times 10^{6}$ \\
Minimum Source-Detector Gap, $d_{\text {min }}$ & $100 \mu \mathrm{m}$ \\
Integration Time, $\tau$ & $1000 \mathrm{~s}$ \\
Detector Area, $A_{d}$ & $1 \mathrm{~cm}^{2}$ \\
Source Thickness, $t_{s}$ & $200 \mu \mathrm{m}^{2}$ \\
Detector Thickness, $t_{d}$ & $200 \mu \mathrm{m}^{2}$ \\
Source Density $(\mathrm{W}), \rho_{s}$ & $19.3 \mathrm{~g} / \mathrm{cm}^{3}$ \\
Detector Density $(\mathrm{Si}), \rho_{d}$ & $2.3 \mathrm{~g} / \mathrm{cm}^{3}$ \\
Temperature, $T$ & $4 \mathrm{~K}$ \\
\hline \hline
\end{tabular}


J. C. Long, Experimental Status of Gravitational-Strength Forces in the Sub-Centimeter Regime, Figure 1.

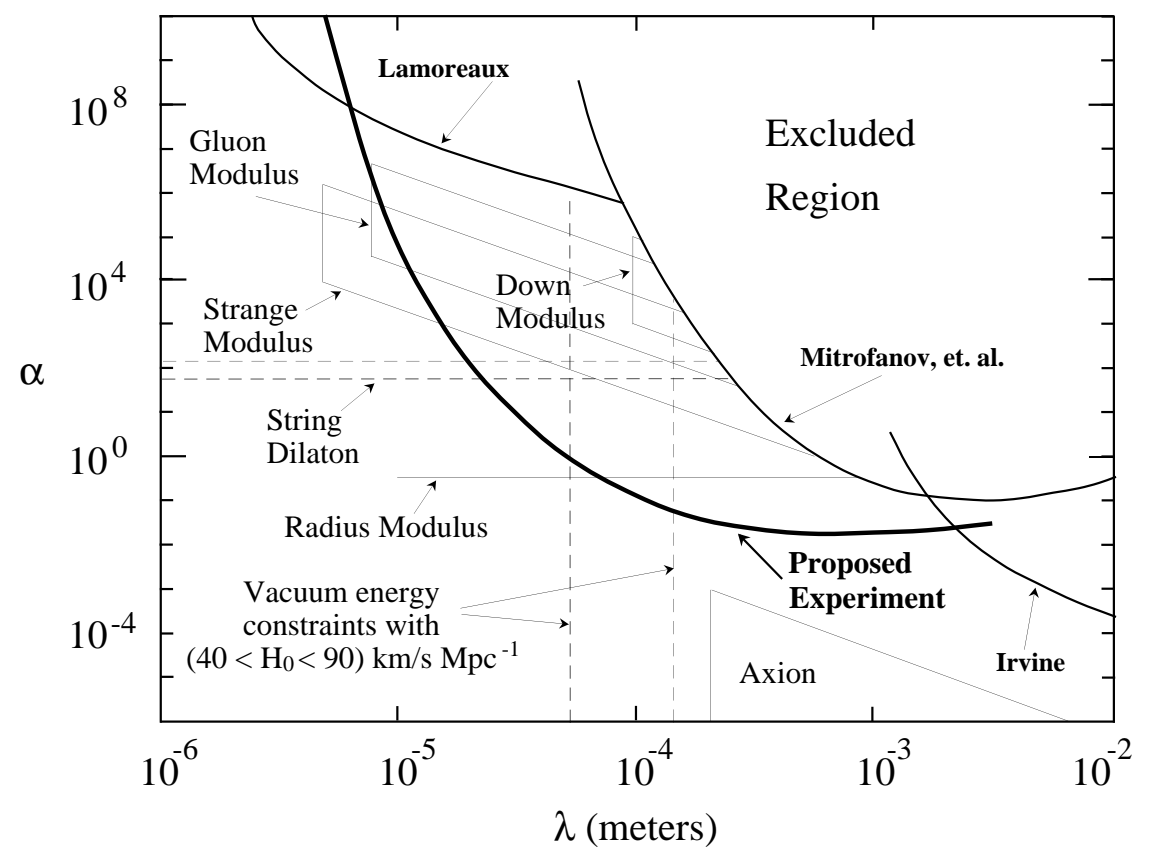


J. C. Long, Experimental Status of Gravitational-Strength Forces in the Sub-Centimeter Regime, Figure 2.

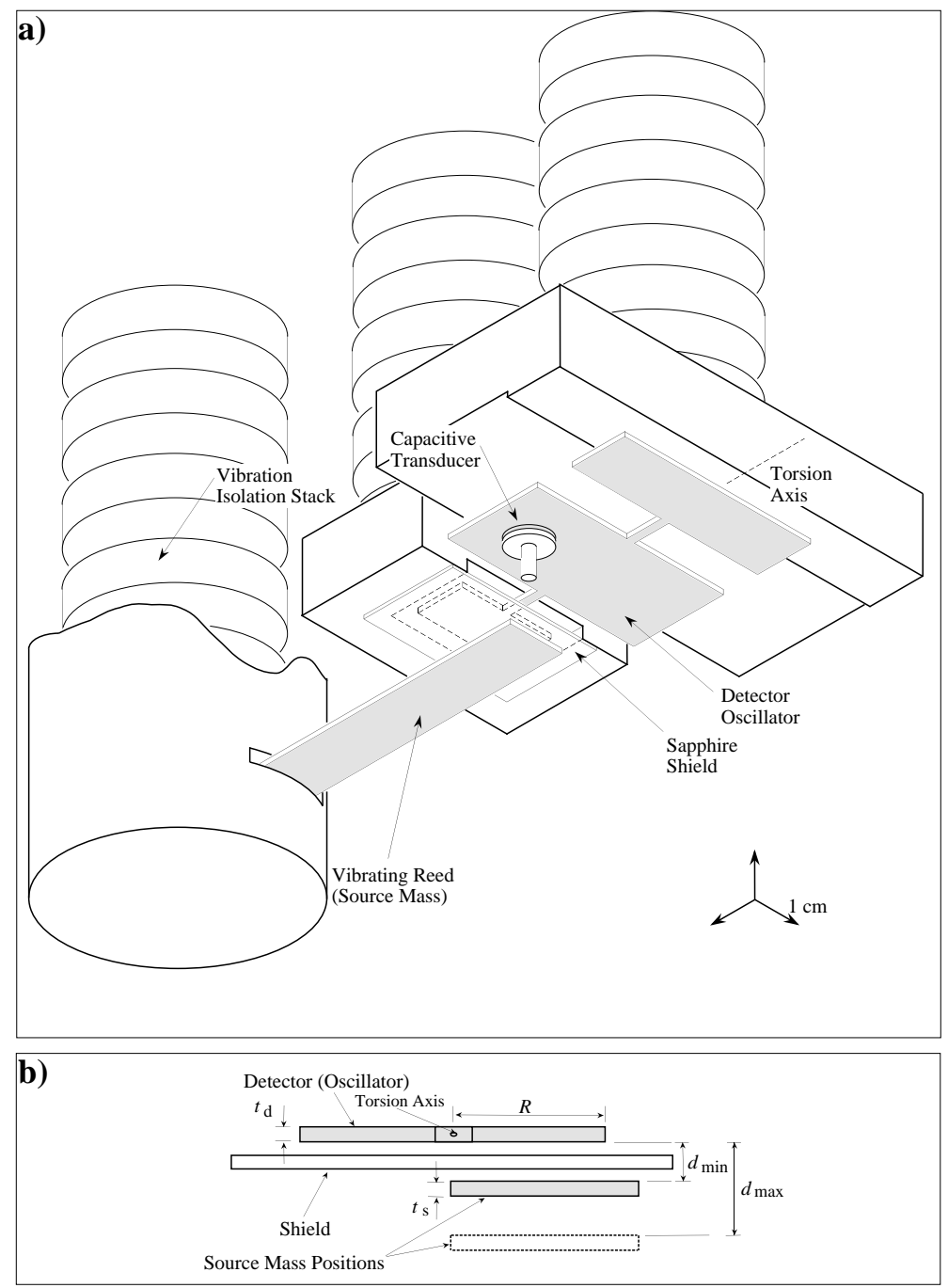

